\title{
Power Domain Multiplexing Approach in MIMO based Non Orthogonal Multiple Access
}

\author{
Sarala Patchala ${ }^{1}$, Dr.M.Sailaja ${ }^{2}$ \\ ${ }^{1}$ Research Scholar, Dept. of ECE, JNTUK, Kakinada \\ ${ }^{2}$ Professor, Dept. of ECE, JNTUK, Kakinada \\ ${ }^{1}$ saralajntuk@gmail.com２maruvada.sailaja@gmail.com
}

\begin{abstract}
The NOMA to achieve with the more number of users in the power domain. NOMA is capable of significantly increasing the number of simultaneous connections. In NOMA the user with precoding data at the base station and reception receives from the radio base station a downlink signal that is non orthogonal multiplied by the same precoding. Here, conventional MIMO system compared with the mm-wave MIMO. The channel sparasity and uncertainty in bema space MIMO system. To reduce inter beam interference in proposed beam space MIMO NOMA system channel vector is determined for each beam to realize pre-coding based on Zero Forcing (ZF). The NOMA achieves higher SE \& EE than OFDMA.
\end{abstract}

Keywords: mmWave, MIMO, NOMA

\section{INTRODUCTION}

The present invention is designed to enable uplink signal transmission power control that is suitable when NOMA is used on the uplink. Joint power optimization problem with to achieve higher spectrum and energy efficient [1]. The existing MIMO system did not concentrate on transmission characteristics. The Multipath environment is placed between the antenna array and user [2]. The conventional MIMO is compared with mmWave MIMO. The existing MIMO need to reduce inter beam interference realize precoding based on zero forcing [3]. Nonorthogonal resource allocation in NOMA indicates that the number of supportable users / devices is not strictly limited by the number of orthogonal resource available. Practical implementation issues in NOMA systems such as its hardware imperfections realizations of massive connectivity [4]. Requirement of channel feedback will be relaxed in power domain NOMA because the CSI feedback is only used for power allocation [5]. Hence there is no need for accurate outdated channel feed back associated with a certain maximum inaccuracy does not change rapidly. NOMA actively investigated in recent years. This may be realized by the sophisticated inter user interference cancellation at the cost of an increased receiver complexity [6]. NOMA has become important principle for the design of radio access technique for $5 \mathrm{G}$ wireless networks.

\section{SYSTEM MODEL} Uplink NOMA
The uplink of NOMA received signal at BS is super imposed signal of $x_{1}, x_{2}$ and $x_{3}$ as follows:

$$
y=h_{1} \sqrt{p_{1}} x_{1}+h_{2} \sqrt{p_{2}} x_{2}+h_{3} \sqrt{p_{3}} x_{3}+W
$$

The UE-User Equipment are 3 consider in this section.

We assume UE-1 is at cell center user, UE-2 cell corner user \& UE-3 cell edge user then no error propagation can be calculated as

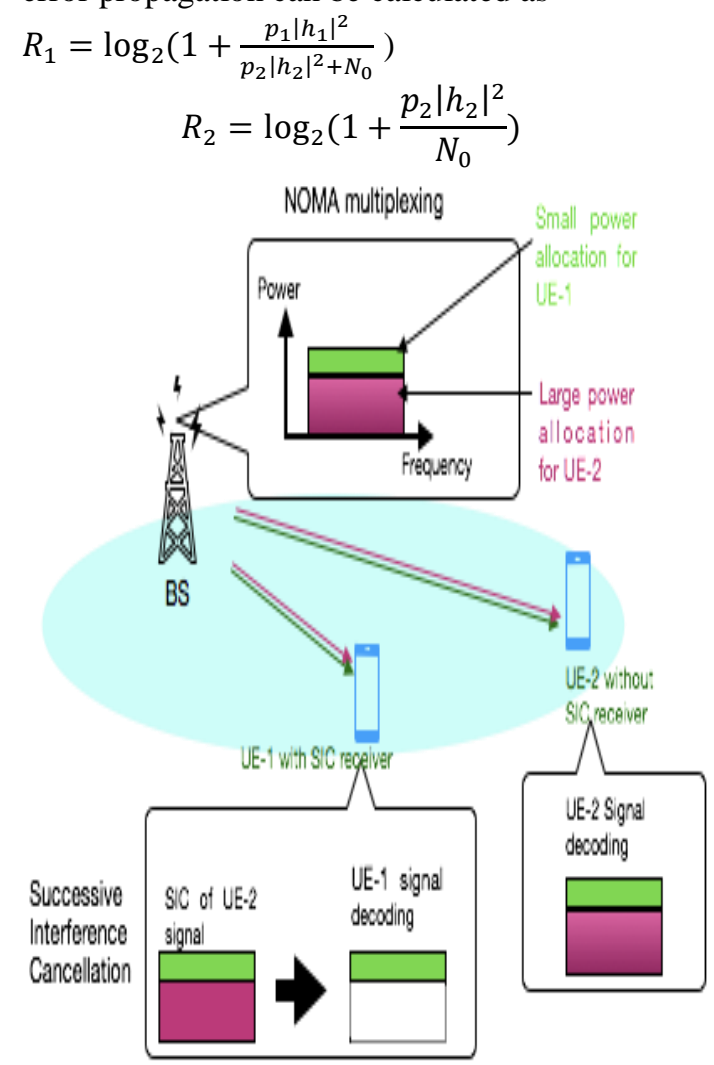

Fig 1. System model 
Now, consider a single cell downlink mm-wave communication system base station with $\mathrm{N}$ antenna $\mathrm{N}_{\mathrm{RF}}$ chain and $\mathrm{k}$ single user antenna simultaneously served by BS [7].

In beam space MIMO

$$
\begin{gathered}
Y=H^{H} W P D+V \\
D=\left[d_{1}, d_{2}, d_{3}, d_{4}, \ldots \ldots d_{k}\right]^{T} \\
D=\left[\begin{array}{c}
d_{1} \\
d_{2} \\
d_{3} \\
: \\
. \\
d_{k}
\end{array}\right]
\end{gathered}
$$

$\mathrm{kx} 1$ transmitted signal vector for $\mathrm{k}$ users.

Table 1. List of access with domain

\begin{tabular}{|l|l|}
\hline Access type & Domain \\
\hline OMA & $\begin{array}{l}\text { Time, frequency and } \\
\text { code access domain, }\end{array}$ \\
\hline NOMA & Power domain \\
\hline Transmitter & Supper position coding \\
\hline Receiver & SIC \\
\hline
\end{tabular}

$$
\begin{gathered}
E\left[D D^{H}\right]=E\left[d_{1} d_{1}^{H}, d_{2} d_{2}^{H}, \ldots . d_{k} d_{k}^{H}\right] \\
=I_{k}
\end{gathered}
$$

Where statistical independent data,

$$
\begin{gathered}
E\left[D D^{H}\right]=E\left[d_{1} d_{1}^{H}\right] E\left[d_{2} d_{2}^{H}\right] E\left[d_{3} d_{3}^{H}\right] \\
E\left[d_{4} d_{4}^{H}\right] \ldots E\left[d_{k-1} d_{k-1}^{H}\right] E\left[d_{k} d_{k}^{H}\right] \\
P=\operatorname{diag}\{p\}
\end{gathered}
$$

Transmitted power all $\mathrm{k}$ users.

$$
p=\left[\sqrt{p_{1}}, \sqrt{p_{2}}, \sqrt{p_{3}}, \ldots . \sqrt{p_{k}}\right]
$$

Satisfies

$$
\sum_{i=1}^{k} \sqrt{p_{i}}=P
$$

Massive MIMO\& NOMA combination gives technical challenges resource allocation. In time and frequency domain improves quality of communication. Massive connectivity dense the network and meets effectively user's QoS requirements. Growth of mobile services are virtual \& augmented reality. mmWave improvement in the spectral efficiency.

Random beam forming does not need to require base stations of the all the users channel vectors. In conventional beam forming random beam forming requires all the users to send the channel vectors.

Stochastic geometry is to apply the sum rate $\&$ outage probabilities. In fast time situations amplitude \& phases of the users channel gain change frequently. The partial CSI on the performance of the mmWaveNOMA downlink network investigated.

The base station set threshold broadcast to the users. Where each user feeding one bit back to the base station it shows quality of channel. In single user case single beam produces with less interference. When compared multi users multiple beam more interference included such as intra interference and inter interference.

The basic principle of NOMA is to simultaneously serve multiple users over same spectrum resources (i.e. time, frequency, code and space) but with different power levels, at the expense of minimal inter-user interference [8].

\section{NOMA DOWNLINK APPROACH}

Now consider a downlink NOMA transmission at BS with single antennas \& single antenna with $m$ number of users with channel gains.

Difficult to realize millimeter wave massive MIMO in practice due to high transceiver complexity and energy consumption. Each antenna in MIMO system requires one radio frequency. The large number of antenna in mm-wave [9] a large number of RF chain. Performance of Beam space MIMO with beam selection is close to optimal. Non Orthogonal Multiple Access (NOMA) with beam space MIMO.

Orthogonal Multiple Access (OMA) relying on the time domain frequency domain and code domain. NOMA realizes in power domain. In this technique [10] the super position coding performed at transmitter and Successive Interference Cancellation (SIC) at the receiver. 


\section{Available online at www.ijrat.org}

New spectrum and energy efficient mm-wave transmission scheme, Beam space MIMONOMA. NOMA is a potential access technique for beam space MIMO in mm-wave communication system.

\section{MmWAVE SYSTEM MODEL}

The mmWave system model is given by $\min \left\{E\left[\left\|\bar{s}-W_{B B}^{H} W_{R F}^{H} \bar{y}\right\|^{2}\right.\right.$

$$
\begin{aligned}
& =\min \| R_{y y}^{\frac{1}{2}}\left(W_{M M S E}\right. \\
& \left.-W_{R F} W_{B B}\right) \|^{2}
\end{aligned}
$$

Where the precoders design $\boldsymbol{F}_{\boldsymbol{B} \boldsymbol{B}} \boldsymbol{F}_{\boldsymbol{R} \boldsymbol{F}}$

Optimum design $\left\|F_{o p t}-F_{R F} F_{B B}\right\|^{2}$

The system model is given by $\bar{Y}=H X$

$$
\begin{gathered}
X=F_{B B} F_{R F} S \\
W_{B B}^{H} W_{R F}^{H} \bar{Y}=\bar{S}
\end{gathered}
$$

Where $W_{R F} W_{B B}$ are the optimization variables. $\min \left\{E\left[\left\|\bar{S}-W_{B B}^{H} W_{R F}^{H} \bar{y}\right\|^{2}\right.\right.$

$$
\begin{aligned}
& =E\{\operatorname{Tr}[(\bar{s} \\
& \left.-W_{B B}^{H} W_{R F}^{H} \bar{y}\right)(\bar{s} \\
& \left.\left.\left.-W_{B B}^{H} W_{R F}^{H} \bar{y}\right)^{H}\right]\right\} \\
& =\operatorname{Tr}\left\{E\left[\left(\bar{s}-W_{B B}^{H} W_{R F}^{H} \bar{y}\right)\left(\bar{s}^{H}-W_{B B} W_{R F} \overline{y^{H}}\right)\right\}\right. \\
& = \\
& \operatorname{Tr}\left\{E \left[\bar{s} \bar{s}^{H}-W_{B B}^{H} W_{R F}^{H} \bar{y} \bar{s}^{H}-W_{B B}^{H} W_{R F}^{H} \bar{y} \bar{s}^{H}+\right.\right. \\
& W_{B B}^{H} W_{R F}^{H} \bar{y} W_{B B} W_{R F} \bar{y}^{H} \\
& =\operatorname{Tr}\left\{E\left[\bar{s} \bar{s}^{H}\right]-E\left[W_{B B}^{H} W_{R F}^{H} \bar{y} \bar{s}^{H}\right]\right. \\
& -E\left[W_{B B}^{H} W_{R F}^{H} \bar{y} \bar{s}^{H}\right] \\
& \left.+E\left[W_{B B}^{H} W_{R F}^{H} \bar{y} W_{B B} W_{R F} \overline{y^{H}}\right]\right\} \\
& =\operatorname{Tr}\left\{R_{s}-R_{s y} W_{R F} W_{B B}-W_{B B}^{H} W_{R F}^{H} R_{y s}\right. \\
& \left.+W_{B B}^{H} W_{R F}^{H} W_{B B} W_{R F} R_{y y}\right\} \\
& =\operatorname{Tr}\left\{-W_{M M S E}^{H} R_{y y} W_{R F} W_{B B}\right. \\
& -W_{B B}^{H} W_{R F}^{H} R_{y y} W_{M M S E} \\
& \left.+W_{B B}^{H} W_{R F}^{H} W_{B B} W_{R F} R_{y y}\right\} \\
& =\operatorname{Tr}\left\{R_{S}-R_{s y} R_{y y} R_{y y}^{-1} W_{R F} W_{B B}\right. \\
& -W_{B B}^{H} W_{R F}^{H} R_{y y} R_{y y}^{-1} R_{y s} \\
& \left.+W_{B B}^{H} W_{R F}^{H} W_{B B} W_{R F} R_{y y}\right\} \\
& =\operatorname{Tr}\{0 \\
& \text { - } W_{M M S E}^{H} R_{y y} W_{R F} W_{B B} \\
& -W_{B B}^{H} W_{R F}^{H} R_{y y} W_{M M S E} \\
& +W_{B B}^{H} W_{R F}^{H} W_{B B} W_{R F} R_{y y} \\
& =\operatorname{Tr}\left\{-W_{M M S E}^{H} R_{y y}^{1 / 2} R_{y y}^{1 / 2} W_{R F} W_{B B}\right. \\
& -W_{B B}^{H} W_{R F}^{H} R_{y y}^{1 / 2} R_{y y}^{1 / 2} W_{M M S E} \\
& \left.+W_{B B}^{H} W_{R F}^{H} R_{y y}^{1 / 2} R_{y y}^{1 / 2} W_{B B} W_{R F}\right\} \\
& =\operatorname{Tr}\left\{A B-B^{H} A+B^{H} B\right\} \\
& =\operatorname{Tr}\left\{(A-B)^{H}(A-B)\right\} \\
& \|A-B\|^{2}=\left\|R_{y y}^{\frac{1}{2}} W_{M M S E}-R_{y y}^{\frac{1}{2}} W_{B B} W_{R F}\right\|^{2} \\
& =\| R_{y y}^{\frac{1}{2}}\left(W_{M M S E}-W_{B B} W_{R F} \|^{2}\right. \\
& W_{M M S E}^{H} R_{y y}^{1 / 2}=A
\end{aligned}
$$

$$
\begin{gathered}
R_{y y}^{1 / 2} W_{R F} W_{B B}=B \\
W_{B B}^{H} W_{R F}^{H} R_{y y}^{1 / 2}=B^{H} \\
\text { Where } E\left[\bar{s} \bar{s}^{H}\right]=R_{s} \\
E\left[\bar{y} \bar{s}^{H}\right]=R_{y s} \\
E\left[\bar{y}^{H} \bar{s}\right]=R_{s y} \\
E\left[\bar{y}^{H} \bar{y}\right]=R_{y y} \\
W_{M M S E}^{H}=R_{s y} R_{y y}^{-1}
\end{gathered}
$$

The transmitter and receiver UE beam Table 2. List of operator

\begin{tabular}{|l|l|}
\hline Operator & Description \\
\hline$(.)^{\mathrm{T}}$ & Transpose \\
\hline$(.)^{\mathrm{H}}$ & Conjugate transpose \\
\hline$(.)^{-1}$ & Matrix inversion \\
\hline$()$. & $\begin{array}{l}\text { Moore pen rose matrix } \\
\text { inversion }\end{array}$ \\
\hline $\operatorname{tr}()$. & Trace of matrix \\
\hline $\operatorname{diag}\{\mathrm{p}\}$ & Diagonal matrix \\
\hline $\mathrm{E}\{\}$. & Expectation \\
\hline
\end{tabular}

pattern sectorial directive gain is given by

$$
G(\varnothing)=\left\{\begin{array}{l}
G_{M}, \emptyset \leq 0 \\
G_{m}, \emptyset>0
\end{array}\right.
$$

where $G_{M}$ is main lobe gain\& $G_{m}$ is minor lobe gain. For Rth mmWave base station is given by $G_{R}=G_{s}\left(\emptyset_{s}\right) G_{U}\left(\emptyset_{u}\right) . \emptyset_{s}$ is the Angle of depature, $\emptyset_{u}$ is angle of arrival. NOMA base station all users are arriavl in terms of power domain only. The noise is assumed as AWGN [11].

The coverage of probability is used to charcterises the quality of network coverage. According to SIC the nth order coverage of probabilty is

$$
\begin{aligned}
P_{n}(T)=\mathbb{P}\left(\operatorname{SINR}_{n, 1}>T, \operatorname{SINR}_{n, 2}\right. \\
>T, \operatorname{SINR}_{n, 3} \\
>T, \ldots \ldots \operatorname{SINR}_{n, n}
\end{aligned}
$$

QTM05G mmwave antenna module is the world's first announced fully Integrated RF for smart phone \& other devices. To improve nnwave signal using 5G technology [12]. The module contain a 5GNR radio transciever, power maientenance, $\mathrm{RF}$ front end phased antenna array. Mmwave improves for wireless communication host challenges like Propagation, range, size. 


\section{Available online at www.ijrat.org}

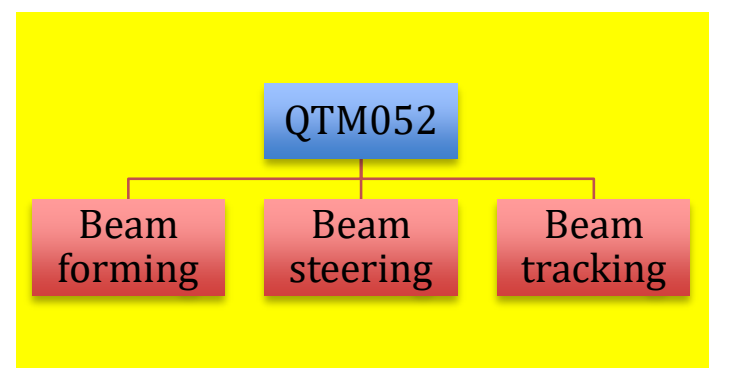

Fig 2: QTM052 Features

The beam forming, steering and tracking for bidirectional mobile mmwave communication sytem drastically improves the range \& coverage of signals [13-16]. Design supports upto MIMO with dual layer polarization for both up \& down link.

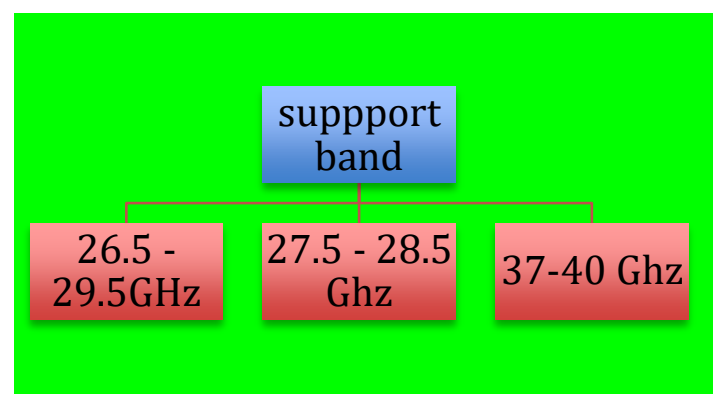

\section{Fig3.Frequency band support}

\subsection{Precoding based Zero Forcing}

Consider zero forcing MIMO channel model $y=H x+n$. Where $\mathrm{y}$ is output system, $\mathrm{H}$ is channel; $\mathrm{x}$ is the input \& $\mathrm{n}$ is the noise. The resulting receiver and cost function are important for the system. The cost function is given by $\widehat{x_{Z F}}=\arg \left\{\min \left[\|y-H x\|^{2}\right]\right\}$. $\|y-H x\|^{2}=(y-H x)^{H}(y-H x)$

$$
=\left(y^{H}-x^{H} H^{H}\right)(y-H x)
$$$$
=y^{H} y-y^{H} H x-x^{H} H^{H} y+x^{H} H^{H} H x
$$

Take Gradient on function, According to the gradient $\operatorname{rule} \nabla()=$.0 . Then the function reduces to

$$
\begin{gathered}
\|y-H x\|^{2}=0-H^{H} y-H^{H} y+2 H^{H} H x \\
=-2 H^{H} y+2 H^{H} H x
\end{gathered}
$$

\section{SIMULATION RESULTS}

In our analysis we assume static power consumption for the network due to power amp add to the power consumed for infoemation waveform. The total power consumption at the transmitter is given by

$$
P_{\text {total }}=P_{T}+P_{\text {static }}
$$

the Energy Efficient is the

$$
\begin{aligned}
E E=\frac{R_{T}}{P_{\text {total }}} & =S E \frac{W}{P_{\text {total }}}(\text { bits } / \text { joule }) \\
S E & =\frac{R_{T}}{W} b p s / \mathrm{Hz}
\end{aligned}
$$

where $\mathrm{SE}=$ Spectrum Efficient

\section{$\mathrm{EE}=$ Energy Efficient}

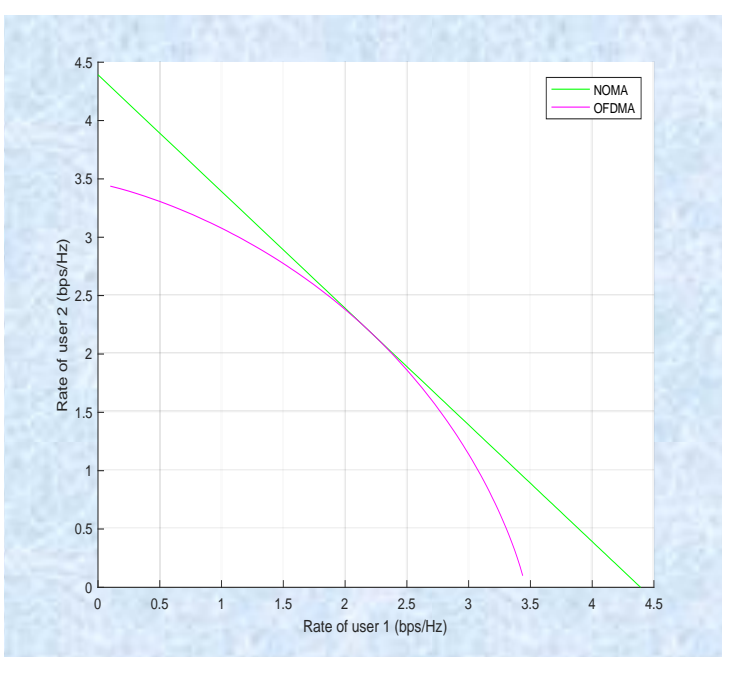

Fig 5.a.SNR1 $=20 \mathrm{~dB}, \mathrm{SNR}=20 \mathrm{~dB}$

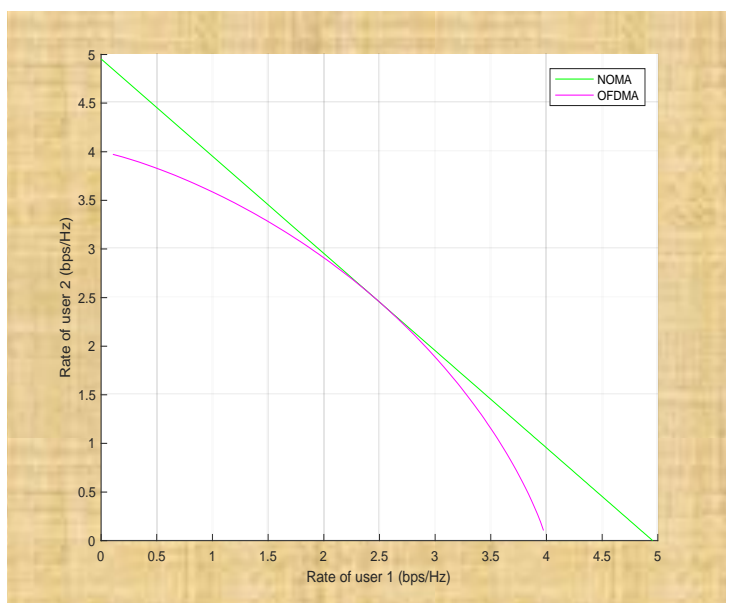

Fig 5.b. SNR1=30dB,SNR2=30dB

\section{Fig 5 rate of user NOMA Vs OFDMA}

In Fig 5 rate of user1 and user2 with NOMA and OFDMA. In Fig 5.a. shows that the SNR1 is $20 \mathrm{~dB}$ and SNR2 is $20 \mathrm{~dB}$. In Fig 5.b. the SNR1 is $30 \mathrm{~dB}$ and SNR2 is $30 \mathrm{db}$. 


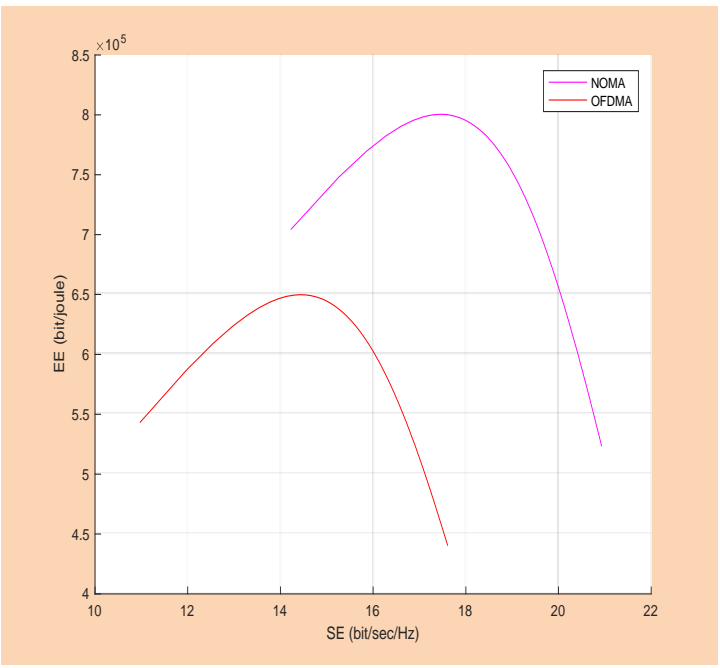

Fig 6.a.

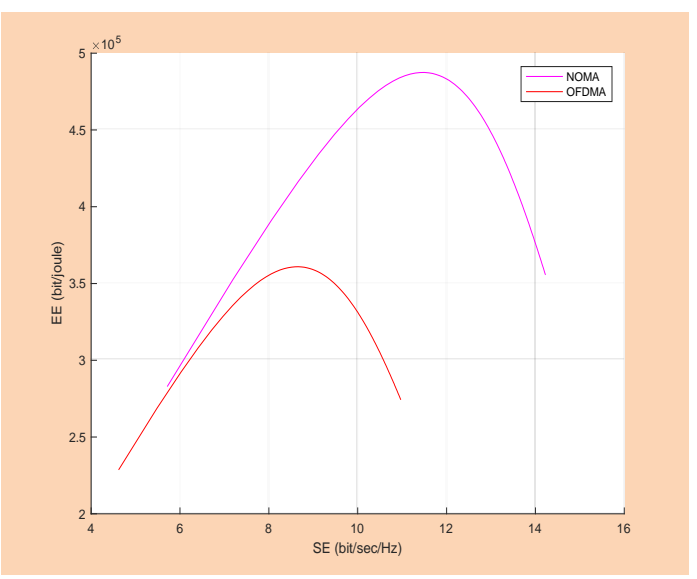

Fig 6.b.

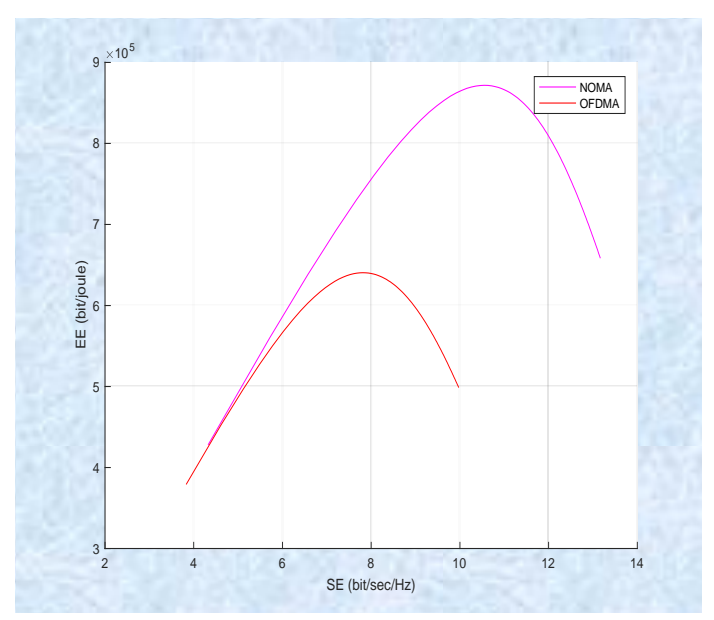

Fig 6.c

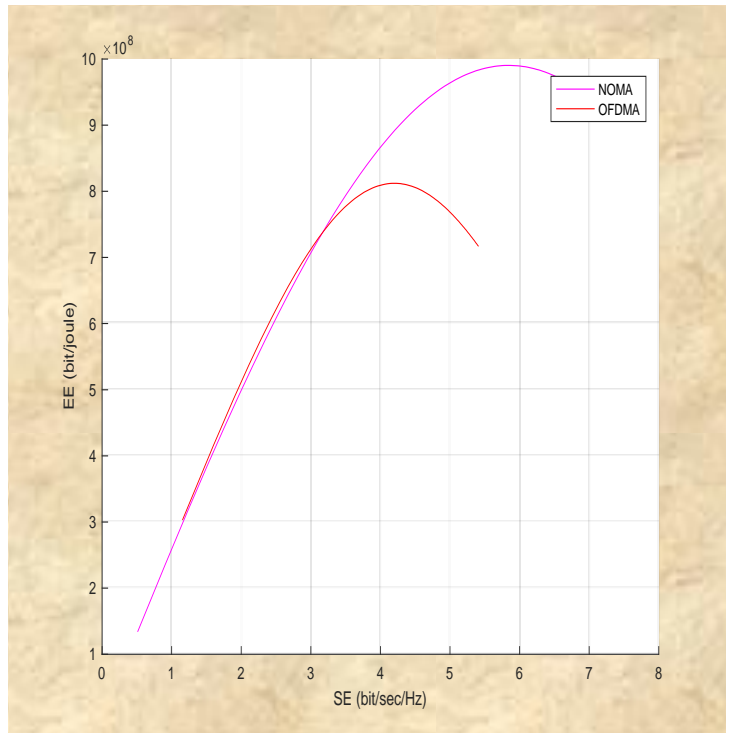

Fig 6.d

\section{Fig6 Energy and Spectral Efficient of NOMA \& OFDMA}

The Fig 6 represents that the energy efficient and spectral efficient of the ssytem for NOMA and OFDMA. In Fig 6.a. Where the badwidth is assumed here $5 \mathrm{MHz}$ and user1 with SNR of $-100 \mathrm{~dB}$ and user2 with SNR of $120 \mathrm{~dB}$. In Fig 6.b. 5MHz with the user1 with SNR of $-120 \mathrm{db}$ and user 2 with $-140 \mathrm{~dB}$. In Fig 6.c. $10 \mathrm{MHz}$ with user1-120 dB and user2 140dB. In Fig 6.d.where $26.5 \mathrm{GHz}$ with the user1-100 dB and user2 -120dB. The NOMA achieves higher SE \& EE than OFDMA.

\begin{tabular}{|l|l|l|}
\hline $\begin{array}{c}\text { Bandwid } \\
\text { th }\end{array}$ & $\begin{array}{l}\text { Modul } \\
\text { ation } \\
\text { index }\end{array}$ & NOMA \\
\hline $20 \mathrm{MHz}$ & 0.5 & $\begin{array}{l}235.70, \\
120.97,114.70\end{array}$ \\
\hline $10 \mathrm{MHz}$ & 0.5 & $\begin{array}{l}129.91 \\
66.53,63.3854\end{array}$ \\
\hline $5 \mathrm{MHz}$ & 0.5 & $\begin{array}{l}70.35 \\
35.96,34.39\end{array}$ \\
\hline $2 \mathrm{MHz}$ & 0.5 & $\begin{array}{l}30.86 \\
15.74,15.11\end{array}$ \\
\hline $1 \mathrm{MHz}$ & 0.5 & $\begin{array}{l}16.44 \\
8.37,8.06\end{array}$ \\
\hline $0.5 \mathrm{MHz}$ & 0.5 & $\begin{array}{l}8.72 \\
4.44,4.28\end{array}$ \\
\hline $0.5 \mathrm{MHz}$ & 0.2 & $\begin{array}{l}7.4 \\
3.77,3.62\end{array}$ \\
\hline $1 \mathrm{MHz}$ & 0.2 & $\begin{array}{l}13.8 \\
7.05,6.74\end{array}$ \\
\hline $2 \mathrm{MHz}$ & 0.2 & $\begin{array}{l}25.58 \\
13.09,12.48\end{array}$ \\
\hline & & \\
\hline
\end{tabular}




\section{Available online at www.ijrat.org}

\begin{tabular}{|l|l|l|}
\hline $5 \mathrm{MHz}$ & 0.2 & $\begin{array}{l}57.22 \\
29.33,27.89\end{array}$ \\
\hline $10 \mathrm{MHz}$ & 0.2 & $\begin{array}{l}103.8153 .22 \\
50.55\end{array}$ \\
& & $\begin{array}{l}84.06 \\
94.14,89.987\end{array}$ \\
\hline $20 \mathrm{MHz}$ & 0.2 & \\
\hline
\end{tabular}

Table3. Based on frequency band

\begin{tabular}{|c|c|c|}
\hline $\begin{array}{l}\text { Bandwid } \\
\text { th }\end{array}$ & $\begin{array}{l}\text { Modul } \\
\text { ation } \\
\text { index }\end{array}$ & NOMA \\
\hline $26.5 \mathrm{GHz}$ & 0.5 & $\begin{array}{l}0.4314,0.0964, \\
0.3351\end{array}$ \\
\hline $29.5 \mathrm{GHz}$ & 0.5 & $\begin{array}{l}0.3482,0.0778, \\
0.2704\end{array}$ \\
\hline $27.5 \mathrm{GHz}$ & 0.5 & $\begin{array}{l}0.4006,0.0895, \\
0.3111\end{array}$ \\
\hline $28.5 \mathrm{GHz}$ & 0.5 & $\begin{array}{l}0.373,0.0833, \\
0.2897\end{array}$ \\
\hline $37 \mathrm{GHz}$ & 0.5 & $\begin{array}{l}0.2213,0.0494, \\
0.1719\end{array}$ \\
\hline $40 \mathrm{GHz}$ & 0.5 & $\begin{array}{l}0.1894,0.0423, \\
0.1471\end{array}$ \\
\hline $26.5 \mathrm{GHz}$ & 0.2 & $\begin{array}{l}0.069,0.0154 \\
0.0536\end{array}$ \\
\hline $29.5 \mathrm{GHz}$ & 0.2 & $\begin{array}{l}0.0557,0.0124, \\
0.0433\end{array}$ \\
\hline $27.5 \mathrm{GHz}$ & 0.2 & $\begin{array}{l}0.0641,0.0143, \\
0.0498\end{array}$ \\
\hline $28.5 \mathrm{GHz}$ & 0.2 & $\begin{array}{l}0.0597,0.0133, \\
0.0464\end{array}$ \\
\hline $37 \mathrm{GHz}$ & 0.2 & $\begin{array}{l}0.0354,0.0079, \\
0.0275\end{array}$ \\
\hline $40 \mathrm{GHz}$ & 0.2 & $\begin{array}{l}0.0303,0.0068, \\
0.0235\end{array}$ \\
\hline
\end{tabular}

Table4: based on supported band

\section{CONCLUSION}

The average received power is under $10 \mathrm{w}$. The results are calculated for radomly distributed data variables. The above results illustrates that the bandwidth for modulation with diffefrent ranges with different modulation index, offset and average transmitted power. The NOMA for user expressed interms of Mbits/sec. Therefore the NOMA achieves higher SE \& EE than OFDMA.

\section{REFERENCES}

[1]. Z.Zhang, Z.Ma,Y.Xiao, M.Xiao,\& etc "Non-orthogonal multiple access for cooperative multicast millimeter wave wireless networks" IEEE J. Sel. Areas Commun. 35, 1794-1808, 2017

[2]. Saito. Y.Benjebbour,A.Kihiyama and etc system level performance evaluation of downlink NOMA IEEE PIMR2013pp611615.

[3]. S.Naribole, E.Knightly, "Scalable multicast in highly-directional60GHzWLANs" IEEE Trans. Netw. 25,

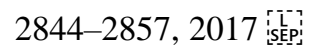

[4]. T. Bai, R.W. Heath, Coverage and rate analysis for millimeter-wave cellular networks. IEEE Trans. Wirel. Commun. 14, 1100-1114, 2015

[5]. J.G. Andrews, T. Bai, M. Kulkarni, A. Alkhateeb, A. Gupta, R.W. Heath, "Modeling and ana- lyzing millimeter wave cellular systems" IEEE Trans. Commun. 65, 403-430, 2017

[6]. Benjebbour, A.,Li, A., Satio, etc system level performance of downlink NOMA for future LTE enhancement IEEE globe com pp66-70

[7] T.S. Rappaport, G.R. Mac Cartney, M.K. Samimi, S. Sun "Wideband millimeterwave propagation measurements and channel models for future wireless communication system design" IEEE Trans. Commun. 63, 3029-3056, 2015

[8]. D.Zhang, Z.Zhou, C.Xu, Y.Zhang, J.Rodriguez, T.Sato, " Capacity analysis of NOMA with siepepmWave massive MIMO systems" IEEE J. Sel. Areas Commun. 35, 1606-1618, 2017

[9]. B.Wang, L.Dai, Z.Wang, N.Ge,S.Zhou, "Spectrum and energy-efficient beam space MIMO- NOMA for millimeterwave communications using lens antenna array" IEEE J. Sel. Areas istepiCOCommun. 35,

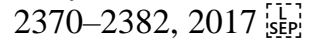

[10]. Sarala Patchala, Dr.M.Sailaja "Reducing of PAPR for MIMO OFDM system” IEEE conference ICECDS-2017, p.p.1475-1478.

[11]. Z.Ding, L.Dai ,R.Schobe \& etc. “ NOMA meets finite resolution analog beam forming in iscepimassive MIMO and millimeter-wave networks" IEEE Commun. Lett. 21, 1879-1882, 2017 is:

[12]. A.J. Morgado \& etc "Hybrid resource allocation iL IEEE Wireless. Commun. 24, 23-29, 2017

[13]. Sarala Patchala, Dr.M.Sailaja "Ergodic channel capacity of multiple input multiple output system" IJARSE ISSN2319-8354, 2017,972-979.

[14]. Sarala Patchala, Dr. M. Sailaja “ Massive MIMO systems" IJRAT45-48

[15]. E.Bjornson, P.Zetterberg, M.Bengtsson, \& B.Ottersten,"Capacity limits and multiplexing gains of MIMO channels with transceiver impairments", 
International Journal of Research in Advent Technology, Vol.7, No.5, May 2019

E-ISSN: 2321-9637

Available online at www.ijrat.org

"IEEECommun.Lett.,vol.17,no.1, pp.9194,2013

[16]. Sarala Patchala, Dr. M. Sailaja "Improvement spectral efficiency in mmWave MIMO system for 5G communications" ICCMC IEEE-2019 $556-560$

\section{Author's Profile:}

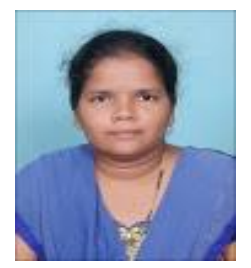

Sarala Patchala, Research Scholor, JNTUK \& Asst.Professor in the deapartment of ECE, KKR \& KSR Institute of Technology \& Sciences, Guntur. Publishing various reputed jounals National \& International. Area of research in wireless communications \& Signal Processing.

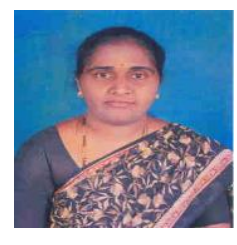

Dr.M.Sailaja, Professor, Department of ECE, JNTUK, Kakinada. Publishing various journals and papers in National and international. Having a experience in teaching and industry. Area of research in communications, networks, computers and etc. 